\title{
Knowledge elicitation in conceptual model building: A case study in modeling a regional Dutch health care system
}

\author{
Jac A.M. Vennix \\ Department of Gamma-Informatics, University of Utrecht, Heidelberglaan 1, 3584 CS Utrecht, Netherlands
}

Jan W. Gubbels

Department of Research Methodology, University of Nijmegen, Thomas van Aquinostraat 4, 6500 HK Nijmegen, Netherlands

Received October 1990; revised May 1991

\begin{abstract}
Client-oriented model building entails eliciting relevant knowledge from the mental models of participants. System dynamicists commonly employ interviews with individuals followed by one or more discussions in freely interacting groups to capture knowledge from a client group. At least two problems arise as a result of this approach. First, it usually demands a high time investment from the client group. Second, freely interacting groups have several drawbacks and are often outperformed by individuals when it comes to generating relevant knowledge. To overcome these difficulties a combination of different techniques for knowledge elicitation is proposed, which is based on useful elements from existing group process methods. The approach consists of three stages with intermediate feedback to participants and can be considered a variant of the Estimate-Feedback-Talk (EFT) approach. Its use is illustrated with a case study of model building in Dutch health care.
\end{abstract}

Keywords: System dynamics, knowledge elicitation, group decision support, mental models, group process technique

\section{Introduction}

Studies evaluating the impact of computer models to support policy making processes in organizations have indicated that client involvement in the model-building process is often a prerequisite for effective model building. One important reason is that the process of model building is frequently more important than the resulting model. Model building itself is largely a learning process about the problem. Most insights about the characteristics of an ill-structured problem are gained during the iterative process of designing a computer model, rather than after the model is finished (House, 1982; Meadows and
Robinson, 1985; De Geus, 1988; Vennix, 1990). Another important reason is that most information in an organization resides in the mental models of organizational members (Forrester, 1987, 1991). Or as Mintzberg puts it: "Thus the strategic data bank of the organization is not in the memory of its computers but in the minds of its managers" (Mintzberg, 1990, p. 166). To support policy making in organizations it is this knowledge which needs to be captured and represented in the model. An important topic in client-oriented or interactive model building thus becomes the elicitation of relevant knowledge contained in the mental models of participants.

Two important problems arise with regard to 
interactive model building. The first is related to the time investment of the client group. In general the process of model building takes a considerable amount of time. And among policy makers and managers time is a scarce resource. The question then becomes how to structure the model building process in such a way that time investment is kept as low as is reasonably possible.

The second problem is related to the sources of knowledge and the techniques to elicit relevant knowledge. System dynamics modelers have primarily relied on interviews with key persons and discussions in interacting groups to capture knowledge from the mental models of participants (Morecroft et al., 1989; Randers, 1977; Richmond, 1987; Richardson and Senge, 1989; Stenberg, 1980; Weil, 1980). Freely interacting groups, however, exhibit several characteristics inhibiting group performance. Among these are the tendency of high-status persons to dominate discussions, inequality of participation and focusing on a single train of thought (Delbecq et al., 1975). Various group process techniques have emerged aimed at improving group performance. Two of the best-known are Delphi (Linstone and Turoff, 1975) and NGT: Nominal Group Technique (Delbecq et al., 1975). Although these techniques contain useful elements, their application to interactive model building is not straightforward. Hence, it is worthwhile to create alternative procedures particularly aimed at knowledge elicitation in system dynamics model building. To guide the design process of such procedures useful elements of existing group process techniques could be used as a basis. In this article we will focus on the design and the implementation of such an alternative procedure. The emphasis is on the design of the procedure and its feasibility. Although we will indicate criteria with which to evaluate the procedure described in this article, we will not primarily be concerned with its evaluation. This will be the topic of future research efforts.

In Section 2 we first review the literature on relevant group process techniques and outline the design of our procedure. In Section 3 we will briefly present the policy problem which is being modeled. In the following sections we discuss each of the stages in our approach in more detail. In a previous article (Vennix et al., 1990) we have given an overall description of this procedure for eliciting knowledge in conceptual model building. In Sections 4 through 6 of this article, we will concentrate in more detail on the central elements in the procedure, i.e. the preliminary model, the questionnaires and the workbooks. These sections are meant to provide the interested reader with enough information to apply this procedure in an interactive model building setting. In a final section we discuss the main results of this study.

\section{Group process techniques for capturing knowledge}

A procedure for interactive modeling and knowledge elicitation will have to meet several requirements. First, the process must be tailored to the iterative character of model building. Second, as compared to approaches including interviews and interacting groups, the process should significantly reduce the participants' time investment. Third, it must allow structured debate on participants' (tacit) assumptions about reality. This is of importance, since most learning takes place during these discussions, because participants share the knowledge contained in their mental models. And finally, the model resulting from this process should not become overly complex. Particularly when the number of participants is large this latter requirement becomes necessary.

With these demands in mind let us take a look at some well-known and relevant group process techniques, i.e. Delphi, Nominal Group Technique (NGT) and Social Judgment Analysis (SJA), in order to establish their utility for interactive model building.

Delphi was originally designed to reduce the inhibiting effects of interacting groups while at the same time preserving the power of pooled knowledge from a group of experts (Dalkey, 1969). This is accomplished by an anonymous procedure employing a series of mailed questionnaires. Results of one iteration are fed back to the panel in the next iteration. The number of cycles is limited by a predetermined criterion, e.g. the level of consensus in the panel or stability in the response patterns. Delphi has been employed numerous times, in particular in studies dealing with technological forecasting. Since the 1970 s a number 
of alternatives emerged. The most well-known of these is the Policy Delphi, which focuses on policy issues rather than on forecasting per se (Linstone and Turoff, 1975).

From the perspective of interactive model building, one advantage of the Delphi method is that time investment for participants is relatively low. Delbecq et al. (1975, p. 29) found that the number of working hours for participants in a Delphi method was one half to one third of those participating in a Nominal Group Technique session or in an interacting group. On the other hand, time and cost for the administrators of Delphi was about twice as much as compared to the other techniques. Another advantage of Delphi is its iterative nature. In terms of elements distinguished by Gustafson et al. (1973) Delphi can be characterized as an iterative EstimateFeedback-Estimate (EFE) process, without faceto-face interaction (i.e. Talk). However, Delphi also has some disadvantages. The conventional Delphi has been harshly criticized by Sackman (1975), among other things because of methodological deficiencies in questionnaire design, the disproportionate emphasis on consensus and the sloppy execution of most Delphi studies. In addition several empirical studies have revealed that Delphi or EFE processes do not outperform interacting groups (Gustafson et al., 1973; Fischer, 1981; Stewart, 1987; Sniezek, 1990). We have to point out, however, that these studies are primarily concerned with estimation and prediction tasks. When it comes to idea generation Van de Ven and Delbecq (1974) found that Delphi significantly outperformed interacting groups and performed almost as well as the Nominal Group Technique. On the other hand, with regard to satisfaction of participants with the procedure NGT clearly scored better than both Delphi and the interacting group. One reason the authors suggest for the lower level of participant satisfaction is the lack of opportunity for clarification of ideas in a Delphi (see also Nelms and Porter, 1985; Van Dijk, 1990). Given our third requirement (i.e. the need for discussion in interactive modeling), the lack of interaction and discussion between participants makes a traditional Delphi less suited for our purposes.

In contrast to Delphi, clarification of ideas and interaction between participants is at the heart of both NGT and SJA. NGT is a procedure to generate and evaluate a number of ideas on an issue with a group of persons joining together in a session. When it comes to generating information (as opposed to evaluating information) numerous laboratory experiments conducted over the last few decades have shown that nominal groups usually outperform interacting groups (Bouchard, 1969, 1972; Lamm and Trommsdorf, 1973; Diehl and Stroebe, 1987). Hence, in NGT the stage of idea generation is strictly separated from the evaluation of ideas. The process consists of the following steps (Delbecq et al., 1975):

- Individuals silently write down ideas.

- Ideas are listed in a round-robin fashion on a flip chart.

- Each idea on the list is discussed for clarification and evaluation.

- Individual rank-ordering or rating of ideas. The group decision is mathematically derived from this voting procedure.

As can be seen from the above sequence NGT basically is an Estimate-Feedback-Talk-Estimate (EFTE) process. Empirical studies have shown that NGT groups (and in general EFTE approaches) outperform interacting and Delphi groups (Gustafson et al., 1973; Van de Ven and Delbecq, 1974; Reagan-Cirincione, 1991) and would thus be useful in interactive model building to improve group performance. In addition the idea of rank ordering and voting on ideas can be usefully applied to identify the most important variables to be included in the model. This will prevent the model from becoming too complex. However, as was the case with Delphi the application of NGT to conceptual model building is neither straightforward. NGT primarily focuses on the listing and evaluation of ideas. Although this is an important aspect the primary emphasis in model building is on structuring ideas. In this sense, reflecting on NGT, Hart et al. (1985, p. 588) call idea structuring the neglected component in group decision making.

In contrast to NGT, which primarily relies on a voting procedure to arrive at a group decision, SJA participants are encouraged to explore the differences in " ... the logic of their underlying judgment policies" (Rohrbaugh, 1979, p. 77). Rather then concentrating on participants' overt opinions the approach focuses on the underlying models participants use to arrive at a decision. This is accomplished by an Estimate-Feedback- 
Talk (EFT) approach (Reagan-Cirincione, 1991). After making an initial individual estimate, participants receive cognitive feedback on the relative weights and function forms each individual applies. Next, differences between individuals in weights and function forms are discussed in an unrestricted manner and the group decision is consensually derived. In two separate studies Rohrbaugh compared Social Judgment Analysis (SJA) with both Delphi and NGT on cognitive conflict tasks of considerable intentional depth (Rohrbaugh, 1979, 1981). In both studies Rohrbaugh found that SJA outperformed Delphi and NGT respectively with regard to individual learning, the reduction of disagreement in the group and the level of satisfaction with the process. For interactive model building the EFT approach as applied in SJA could be extremely useful particularly because it focuses on the systematic discussion of assumptions underlying decisions. An obvious disadvantage is that an iterative EFT procedure would be very time consuming in a complex task like model building.

Summarizing we come to the conclusion that an EFT approach as applied in SJA is useful for interactive model building. Applying this approach to model building would result in a Conceptualize-Feedback-Talk process with one or more iterations, to meet the first requirement. In such a sequence reduction of the time investment for participants, our second requirement could be achieved in two ways. First, rather than to start from scratch, by constructing a preliminary model (Hart et al., 1985) which can be adapted by the client group. Second, by including elements from Delphi, which reduces working hours for participants as we have seen. In addition mailed questionnaires, in comparison with interviews, might also reduce the time investment for the modeler. To satisfy the third demand, one or more group sessions will have to be organized, in which participants can discuss their opinions and ideas. For this purpose in SJA function forms are graphically displayed for discussion. In contrast we primarily rely on causal diagrams to aid and structure discussions. Finally, to meet the fourth requirement (preventing the model from becoming too large) rank-ordering procedures like in NGT could be incorporated in the process.

The approach employed in this case study is based on the above ideas and consists of several stages. After a preliminary definition of the policy problem, in the first stage a small project group constructs a preliminary conceptual model based on a review of the relevant literature and insights within the project group (see also Hart et al., 1985). This preliminary model is used as a basis for the second stage in which the actual participation of the client takes place. In the first cycle of this second stage we employ a questionnaire. The questionnaire aims at eliciting comments from the participants with regard to the significance of concepts and relationships between concepts employed in the preliminary model. The questionnaire is followed by a socalled workbook (Underwood, 1984) in the second cycle. This workbook provides feedback about the results of the questionnaire. In addition it invites participants to comment on more complex submodels constructed by the project group on the basis of the information generated in the questionnaires. Both the questionnaire and the workbook are filled out by participants working individually at home. In the third cycle we employ a structured workshop. In this workshop participants discuss their comments on the workbook's submodels in more detail. In this sense the first two cycles serve a focusing function: they identify those elements in the preliminary model on which participants do not agree. In sum the three cycles lead to considerable adaptation of the preliminary model, which results in a final conceptual model. The above mentioned stages are visualized in Figure 1.

Before discussing each of the stages in more detail we will first briefly introduce the policy problem.

Figure 1. Stages in knowledge elicitation 


\section{The policy problem}

The model is designed for the Health Care Insurance Organization (HCIO) at Zwolle in the Netherlands. The project group consists of two $\mathrm{HCIO}$ health care planners and two experienced system dynamics modelers. In addition some five $\mathrm{HCIO}$ staff members assisted in assessing the preliminary model, the questionnaire, the workbook and the workshops.

The policy problem which is modeled is related to the gradual but persistent rise in health care costs. From 1968 to 1985 total health care costs in the Netherlands increased from about $6 \%$ of the net National Product to about $10 \%$. Studies explaining this rise in health care costs have in general primarily focused on exogenous factors (Grünwald, 1987). Little attention has been given to processes within the health care system which might contribute to rising health care costs. Particularly these endogenous factors, however, might provide policy makers and planners with clues on how to control health care costs in the future. This latter perspective forms the basis for this project. The problem definition for the model-building project consists of three related questions.

a) What factors have been responsible for the increase in health care costs in the past?

b) How will health care costs develop in the future?

c) What are the potential effects of several policy options to reduce these costs?
The above three questions guided the design of the preliminary model by the project group.

\section{The preliminary model}

The process of designing the preliminary model was started by a two-hour brainstorming session within the project group in which a flow diagram of the system was constructed. The diagram is shown in Figure 2.

Persons with health complaints initially consult their general practitioner (g.p.), who decides whether a patient

- has to return (order a patient back);

- will be referred to a medical specialist;

- will be discharged.

The medical specialist, in turn, decides whether his patients

- have to return (order back);

- will be discharged;

- will get a renewed referral from the general practitioner (which is necessary after one year of treatment by a medical specialist);

- will be admitted into hospital and when they will be discharged from hospital.

Health care costs for the general practitioner are in general generated by his decision to perform certain required medical treatments or to prescribe one or more drugs. With regard to medical specialists health care costs are generated by a decision to (re)examine a patient, apply medical surgery (medical transactions) and/or



Figure 2. Patients' flow in the health care system 
Table 1

Potential factors affecting decisions in patients' flow model ${ }^{\text {a }}$

\begin{tabular}{|c|c|c|c|}
\hline consultation & order back g.p. & $\begin{array}{l}\text { refer to } \\
\text { specialist }\end{array}$ & $\begin{array}{l}\text { order back } \\
\text { specialist }\end{array}$ \\
\hline $\begin{array}{l}\text { - patient's age } \\
\text { - \% of women } \\
\text { - (perceived) severity } \\
\text { of complaint } \\
\text { - duration of complaint } \\
\text { - g.p.'s view of job } \\
\text { - g.p.'s view of patients } \\
\text { - urbanization }\end{array}$ & $\begin{array}{l}\text { - patient's age } \\
\text { - checking patients } \\
\text { - chronical disease } \\
\text { - g.p. 's workload }\end{array}$ & $\begin{array}{l}\text { - patient's age } \\
\text { - \% women workload } \\
\text { - chron. disease } \\
\text { - patient's pressure } \\
\text { to be referred }\end{array}$ & - specialist's \\
\hline admit into hospital & no. of prescr. by g.p./spec. & $\begin{array}{l}\text { no. of medical transactions } \\
\text { by spec. }\end{array}$ & cost of medical transactions \\
\hline $\begin{array}{l}\text { - patient's age } \\
\text { - specialist's view of job } \\
\text { - no. of beds }\end{array}$ & $\begin{array}{l}\text { - patient's age } \\
\text { - workload }\end{array}$ & $\begin{array}{l}\text { - spec. view of job } \\
\text { - patient's age }\end{array}$ & - patient's age \\
\hline
\end{tabular}

a Source: adapted from Poppen (1987).

prescribe a drug. Most of these decisions have a quantity and a cost component. For instance: the number of prescriptions by a general practitioner or a specialist (quantity) and the price of the drug prescribed (cost).

The next step is to identify a number of factors which affect the decisions discussed above and to include these in the flow model of Figure 2 to form a hybrid diagram as suggested by Richardson and Pugh (1981). A literature search was carried out to find relevant factors which could be used in developing the preliminary model. Table 1 summarizes the results.

Interestingly enough, as can be seen from the table, most studies concentrate on the 'forward' flow process, i.e. consultations, referrals and admissions into hospital. Almost no research was found on factors affecting flow processes in the opposite direction, i.e. discharges from the general practitioner, the medical specialist and from hospital. Hence, we decided to first concentrate on this 'forward' flow process in the construction of the preliminary model. Most of the variables in Table 1 were used in this construction process. Variables which could not be causally related to the decisions of the actors in the system were left out (e.g. urbanization), which is not to say that these could never be incorporated in the model. As we will see in the next section, participants are invited to add factors to the preliminary model which they consider important. In this respect it is important to point out that no effort was made by the project group to make this preliminary model perfect, since it primarily serves a 'trigger' function to start the knowledge elicitation process. It was argued that a 'perfect' preliminary model would hardly be motivating for participants and would most probably not give them a feeling of 'ownership' over the conceptual model. The preliminary model is shown in Figure 3.

The model shown in Figure 3 served as a basis for the first step in the knowledge elicitation process: the questionnaire.

\section{The questionnaire}

In any interactive model building process one has to decide on at least two issues, i.e. the selection of participants and the method to elicit relevant knowledge. To avoid receiving one-sided, biased information we incorporated a variety of persons with different backgrounds. Our participants belong to various organizations in three fields, namely the actual care system (e.g. general practitioners, medical specialists), the policy making field (e.g. planning institutions) and the social behavioral research field (e.g. university health care research units). Participants were selected in a two-step procedure. First, relevant organiza- 




Figure 3. Preliminary conceptual model

tions were listed. Next, within these organizations we identified some sixty potential participants, fairly well spread over the three fields.

We took several precautions to avoid low response. For example, we enclosed with the questionnaire an abstract of an article written on the construction of the preliminary model. In addition we pointed out to the respondents that we needed their expert opinion in order to be able to improve the preliminary model. These precautions paid off. The response rate exceeded $95 \%$, which is very high for a mailed questionnaire.

The second issue is the questionnaire design. In order to elicit causal arguments we took as our point of departure the binary relationships in the preliminary model and translated these into verbal statements. For instance, in Figure 3 the accompanying verbal statement for the arrow 
running from 'mean age of patients' to 'refer' is: "Older patients are referred more often to a medical specialist than younger patients".

First we asked participants whether they agreed, partially agreed or disagreed with the statement and second we invited them to indicate why they agreed or disagreed. Naturally, from the point of view of knowledge elicitation this second part of the question is the most interesting, since it provokes causal arguments from the respondent's mental model. For example, most respondents agree with the above statement presenting explanations like:

- older patients have more and more serious complaints,

- they have more pathology,

- the chances of serious pathology are much bigger,

- resulting in more polypathology,

- polypathology, therefore a more complex diagnosis, and thus more complex therapy, etc.

The task of the project group is twofold. First, if possible, to combine concepts employed in these arguments into a smaller number of categories. Second, to derive a causal structure from these argumentations. In order to accomplish this a couple of persons from the project group conducted a qualitative content analysis and made initial suggestions which were in turn checked by the other two persons from the project group. Discussions in the project group then led to a final decision for each of the statements. From the argumentations presented on the above statement for instance, the project group derived the following causal argumentation: "Older patients frequently have more polypathology. This impedes a correct diagnosis, hence a specialist's opinion is needed, which causes older patients to be referred more often". Rather than the two original variables (average age and number of referrals) one now has four variables related into a causal chain: average age, polypathology, complexity of diagnosis and number of referrals. In the second cycle (workbook) this elaborated argument is reported back to the participants and they are invited to indicate whether they agree with this formulation or not.

The example presented above is a clear and one of the most straightforward examples of eliciting causal arguments from the participants' mental models. This does of course not happen in all cases. Sometimes no argumentations are presented or it is pointed out that the statement itself is obvious.

However, causal argumentations are not the only type of interesting information which can be derived from the questionnaire. Other interesting conclusions from the 'why' part of the questions were related to the concepts themselves. Take for instance the following statement: "The higher a general practitioner's workload the higher the number of prescriptions". Approximately half of the respondents (partially) agreed while the other half did not. This is of course quite confusing. To resolve this apparent contradiction the project group made two lists of argumentations: one containing the argumentations of those who agreed and one containing the argumentations of those who did not agree. Careful content analysis and comparison of arguments provided by advocates and opponents revealed that the two groups did not use the same concept. One group obviously had the temporary rush during the consulting hour in mind (for instance caused by an epidemic of influenza). The other group presented arguments which were related to structural workload. Interpretations like these made by the project group were also reported back in the workbook and participants were asked to comment on it. A similar example on the concept 'general practitioners view of his job' will be discussed in the next section when we show a sample of part of the workbook.

The questionnaire was divided in a number of sections each dealing with one 'dependent' variable, e.g. number of referrals or number of prescriptions by general practitioners. Each of these sections thus contained a number of statements together with 'why questions'. At the end of each section we asked respondents to add variables (affecting the dependent variable) which were not included in the preliminary model. As will be clear, this results in quite a number of factors, which cannot all be included in the model. Hence the last question in each of the sections to indicate the three most important factors. A sample of the questionnaire is presented in Figure 4.

From the last question in each section we calculated frequency distributions and the three factors mentioned most frequently as important 
were used to develop submodels around a dependent variable in order to carry the conceptual model building process one step forward.

\section{The workbook}

In order to be able to develop more complex submodels and have these criticized by the partic-

\section{Introduction and explanation.}

...

Section 1: consulting the general practitioner

...

Section 2: ordering patients back by general practitioners

\section{A. Statements}

1. The higher a general practitioner's workload the more patients he will refer to a specialist

agree

partially agree

disagree

because

2. The broader a general practitioner's view of his job, the more patients he will order back

0 agree

partially agree

o disagree

because

3. etc.

B. Considering the number of referrals by general practitioners do you think there are any other factors, apart from the ones mentioned above, which affect the number of referrals?

C. Which three of the above mentioned factors (including the ones you added in the previous question) do you consider most important in explaining the number of referrals? Please indicate the most important first etc.

1

2

3

Section 3: referrals by general practitioners

...

Section 4: prescriptions by general practitioners ipants we employed a so-called workbook in the second cycle. The workbook consisted of about 30 pages (including diagrams and space for comments). It is a kind of questionnaire with a particular format. In the workbooks we explained in more detail the process of model building and the diagramming tools, we fed back the results of the questionnaire and again we invited participants . $\ldots$ 


\section{Consultations by patients}

...

...

\section{Backorders by the general practitioner}

In the previous section we focused on the decisions of patients to consult their general practitioner. In this and the next two sections we discuss three decisions of general practitioners: ordering patients back, prescribe drugs, refer to a medical specialist or combinations of these. In this section we focus on factors affecting the process of ordering patients back.

\subsection{Results of the questionnaire}

From the questionnaire we conclude that with regard to the number of patients ordered back by a general practitioner the uncertainty of the general practitioner is considered the most important factor. About $90 \%$ of the respondents agrees with the statement that more uncertainty leads to more patients being ordered back. From the argumentations presented with the statements, however, it turns out that the statement cannot be maintained in its current form. We will refer back to this in section 2.2 of this workbook. The second most important factor is the general practitioner's view of his job (about $75 \%$ agrees with this statement). This statement too will have to be elaborated as we will see in Section 2.3 of this workbook. The third most important factor is the general practitioner's workload. We will discuss this in Section 2.4 .

\subsection{A general practitioner's uncertainty}

Most respondents indicate that uncertainty leads to more control behavior, which in turn increases the number of patients ordered back. There is a problem however. A number of respondents states that more uncertainty can also lead to more referrals to medical specialists. In our opinion this depends on the aspect about which a general practitioner is uncertain. We distinguish three kinds of uncertainty, i.e. uncertainty with regard to:

- the diagnosis,

- the expected progress of the disease,

- the potential effect of the therapy.

In our opinion the first kind of uncertainty will lead to either more referrals or more prescriptions or both. We will come back to that in Chapters 3 and 4 of this workbook. The second and the third kind of uncertainty of a general practitioner will lead to more patients being ordered back. We formulate the following statements:

1. The more often a general practitioner is uncertain about the expected progress of the disease, the more often he will order patients back.

2. The more often the general practitioner is uncertain with regard to the effects of the therapy, the more often he will order patients back.

A number of respondents points out that the uncertainty of the general practitioner will decrease with his number of years of experience. Some assume that this is amongst others related to the fact that he will know more about his patients. Hence:

3. The more experienced a general practitioner is in his profession, the better he is informed about the history/background of his patients and the less uncertain he will be.

Using a causal diagram the above statements can be visualized as below.

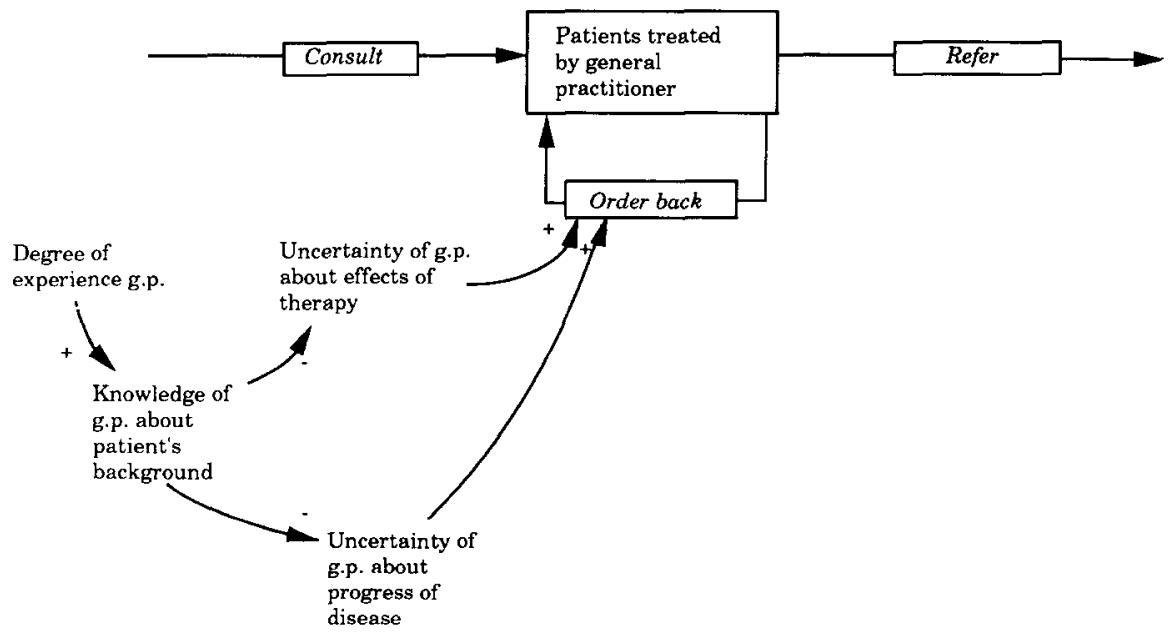

Figure 5. Sample of the workbook used in knowledge elicitation 
Task 1: Please indicate in this diagram with which parts you do not agree by crossing these out. Please write down any comments in the space below.

\subsection{The general practitioner's view of his job}

The general practitioner's view of his job also leads to some differences of opinion between respondents. Most probably this is due to a difference in the interpretation of the concept of 'view of his job' (as was the case above with uncertainty). Those respondents agreeing with the statement that a broad view of the job will lead to more patients being ordered back argue that a general practitioner with a broad view will carry out more therapeutic and diagnostic transactions and will check more patients himself (rather than refer to a specialist) and hence will order back more patients. These general practitioners will also refer less in those respondents' opinion. Those respondents who do not agree with the statement point out that a general practitioner with a broad view will provide better and more specific aid to his patients which will lead to less patients being ordered back. This contradiction between the two groups can in our opinion be explained from the fact that different persons interpret the concept 'view of job' in different ways. One group considers 'view of job' as the number of tasks that a general practitioner considers to be part of his job. The other group seems to interpret the concept as the way a general practitioner handles his patients. From here on we will define 'view of job' as the number of tasks the general practitioner considers to be part of his job. The way a general practitioner handles his patients will be denoted by the concept 'g.p.-patient relationship'. Below we will first focus on the g.p.-patient relationship.

From the comments in the questionnaires we can derive a few factors which are considered important in this respect, i.e. the quality of the communication during consultation and a patient's confidence in his general practitioner. We formulate the following statements about the, 'g.p.--patient relationship':

4. The more susceptible the general practitioner is to the patient's complaint, the higher the quality of the discussion during consultation.

5. If the quality of the discussion during consultation increases the patient will get more confidence in his general practitioner.

6. The more confidence a patient has in his general practitioner, the more information (quantitatively and qualitatively) he will provide about his complaint to the general practitioner.

7. The more information a patient provides, the higher the quality of the discussion during consultation.

8. The higher the quality of the consultation discussion, the less a general practitioner will order patients back.

Adding these factors to the previous figure results in the figure below.

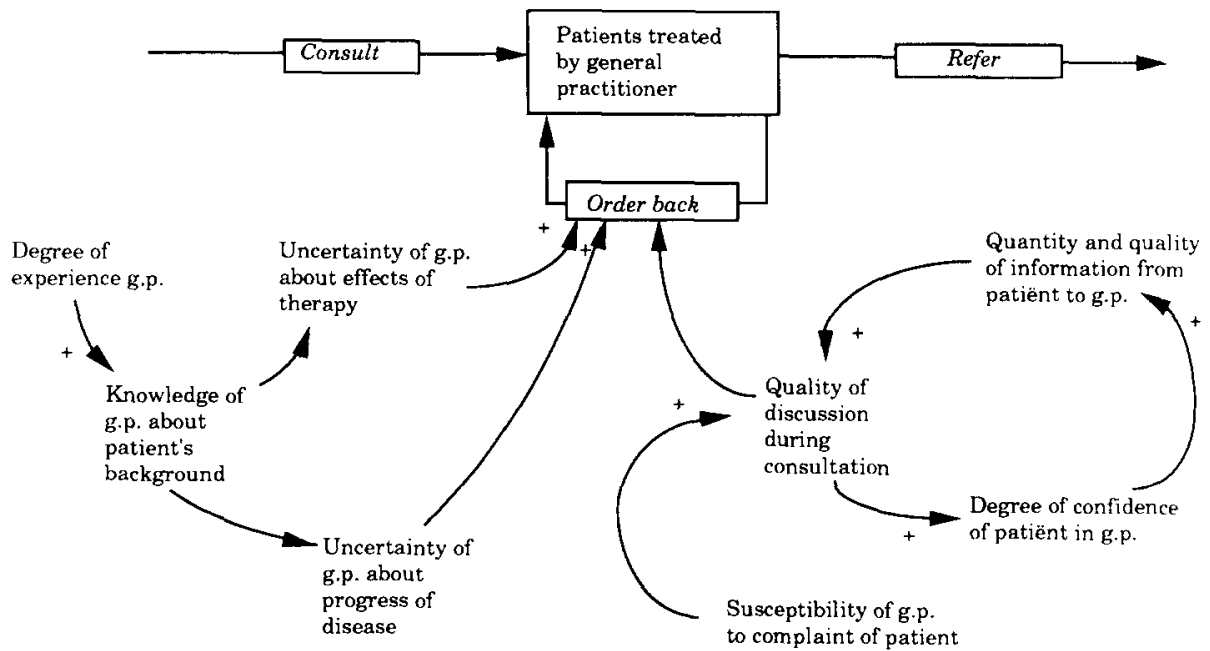

Figure 5 (continued). Sample of the workbook used in knowledge elicitation

to comment on the submodels developed in the workbook.

The workbook was also meant to prepare the participants for the third stage in knowledge elici- tation: the structured workshop. Two subsets of 9 respondents (from the original 60 ) were selected to fill out the workbooks and to participate in one of two workshops. We selected 18 participants 
Task 2: Please indicate in this diagram with which parts you do not agree by crossing these out. Please write down any comments in the space below.

2.4 A general practitioner's workload

...

$\ldots$

\section{Prescriptions by general practitioners}

-..

$\cdots$

4. Referrals by general practitioners

$\cdots$

Figure 5 (continued). Sample of the workbook used in knowledge elicitation

spread over the three fields mentioned above (i.e. actual care system, policy making organizations, research institutions) who presented us with the most detailed comments and argumentations in the questionnaires. All 18 filled out the same workbook. The workbooks and the two work- shops both covered that part of the model which is related to the first echelon (general practitioners), since a number of medical specialists (the second echelon) refused to cooperate because of a conflict between their interest group and the central government.

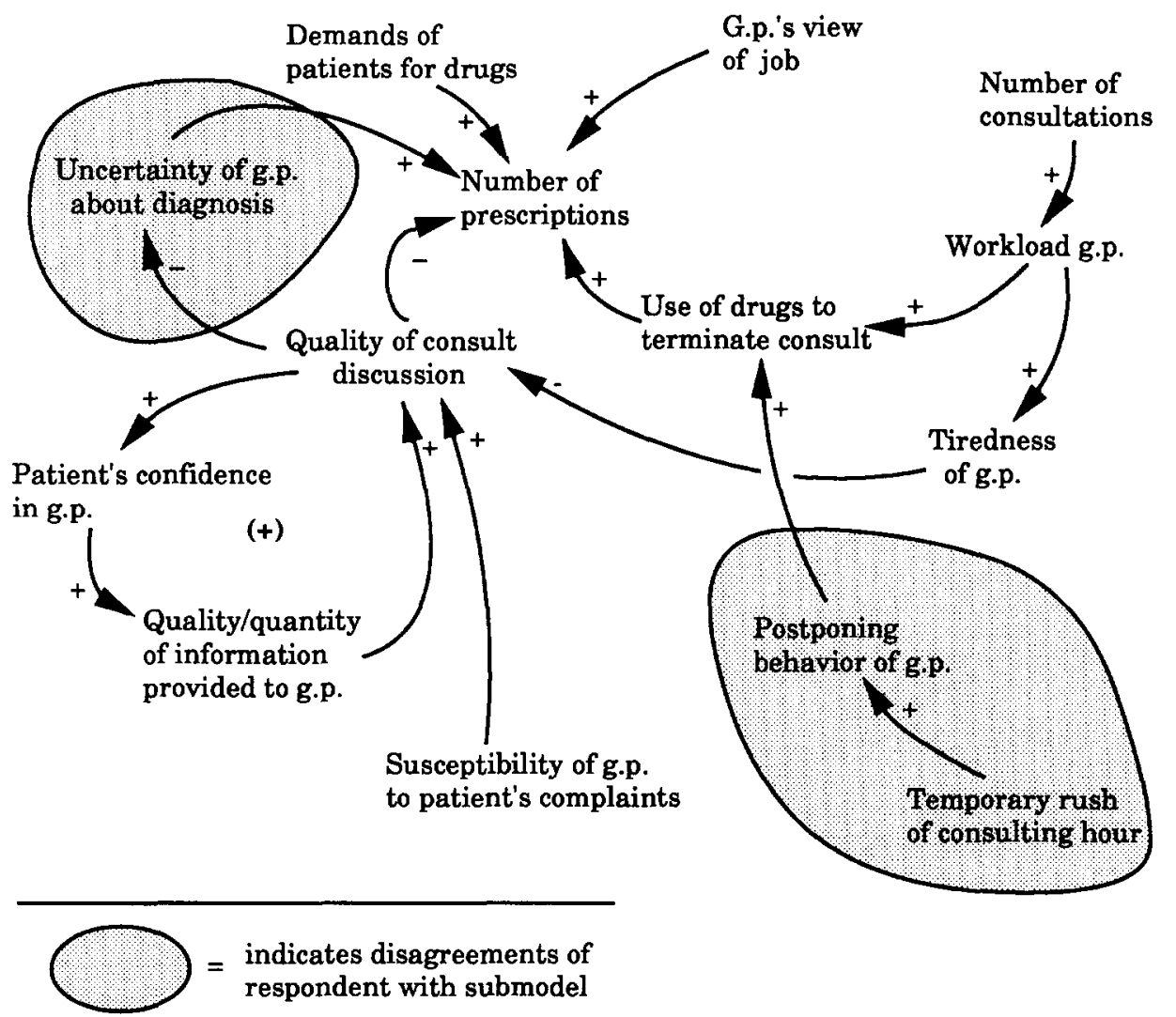

Figure 6. Conceptual submodel on 'number of prescriptions' from workbook 
The workbook contained four submodels, centered around four important 'dependent' variables in the preliminary model, i.e. consultation by patients on the one hand and prescriptions of drugs, referrals and 'back orders' by general practitioners on the other. These four submodels were developed by the project group using the preliminary model and the results of the questionnaires. This does not mean however that the design of the submodels was straightforward. Although the questionnaires provided us with the three most important variables affecting each of the above 'dependent' variables and with intermediary links between two variables, this information was not always sufficient to produce a submodel. Hence, the project group frequently had to fill in 'causal gaps' between these dependent and independent variables where the questionnaires did not provide that information. Again research literature was consulted and discussed within the project group to generate the necessary information.

In order to simplify matters for the participants the submodels are built up gradually in the course of the workbook. This was accomplished by first linking the most important independent variable (mentioned in the questionnaire) to the dependent variable. Next, variables were identified which could explain this independent variable and so on until a network of causal relationships was constructed. The verbal explanations were summarized by means of a causal diagram, in which the participant could indicate his comments and suggestions for adaptations. The same procedure was followed with regard to the second and third most important variable. In order to illustrate the procedure used in the workbooks, we have reproduced part of the workbook (on the phenomenon of ordering patients back) in Figure 5.

Once a submodel was complete, the respondent was invited to summarize his comments by indicating his disagreements with the submodel as shown in Figure 6 . He then had to continue with the next submodel.

The completed workbooks were sent to the project group one week before the workshop. They were used to determine the topics for discussion and to organize the subgroups.

\section{The structured workshop}

For the actual design of the workshop we relied on our experience with previous workshops and guidelines found in the literature (e.g. Duke, 1980; Hart et al., 1985; Mason and Mitroff, 1981; Vennix and Geurts, 1987). In addition we employed a few conclusions from the research literature on small groups. One is that introduction of structure in group activities drastically improves group performance (Bouchard, 1969). Another is that participation can be improved by using small task groups (Eden, 1985; Hart et al., 1985) and a group facilitator to structure plenary discussions.

There were also some impediments that we had to take into account. For examples since general practitioners participated in the workshops these could not be held during daytime. Hence we started at 4 p.m. and had to be finished by 9 p.m.

In order to use the available time as efficiently as possible and to improve participation in the discussions we formed three task groups of three persons to allow in-depth discussions of different submodels during the workshop. Task groups were composed of persons with similar comments on the submodels. Each of the three task groups discussed one of the submodels. From the four submodels in the workbook we selected the three that received most criticism in the workbooks. The program for the workshop was as follows:

$4.00-4.15$ p.m.: welcome to participants.

4.15 - 5.00: introduction and explanation.

$5.00-6.00$ : $\quad$ task group discussions.

$6.00-7.30: \quad$ plenary session.

$7.30-8.15: \quad$ dinner.

$8.15-9.00$ : discussion on feedback loops.

$9.00-9.15$ : evaluation and conclusion.

In order to facilitate work in the subgroups each was assisted by one member of the project team. To structure subgroup activities we used a few aids. First, each group member was assigned a role with accompanying responsibilities. For instance, one person was responsible for time management, another for presentation of the results of the subgroup discussions in the plenary session. Second, to feed back the results from the workbook and as a potential starting point for discussion we provided each group with a copy of 
the submodel diagram. On these diagrams we indicated by means of different colours which person had criticized what part of the submodel (see also Figure 8 ). The diagrams provided to the participants were used as a kind of scribbling paper during discussion about the submodels. The diagrams could be modified by participants as they saw fit. In addition we returned the workbooks to participants as an aid in the discussion.

At the end of the task group session one person recorded the final changes in a large format diagram, which was put on the wall in the plenary session room. The spokesperson of the first task group was then given ten minutes to explain the changes in the submodel. After answering any clarifying questions, there was a 20 minute plenary discussion about the submodel. This procedure was repeated for the other two submodels. After the break for dinner there was a discussion on the notion of feedback loops that could be identified within the model.

Participants were quite involved in the discussions and were very satisfied that there was a clear time table, which was followed quite strictly. Although there was consensus on many issues, it also became clear that several processes in health care are poorly understood. Here the knowledge elicitation process was arrested at the point where there were only vague conjectures. This was for instance true with regard to the number of 'back orders' by a general practitioner. Lack of knowledge on general practitioners' back orders is largely due to disinterest of the insurance companies (as far as mandatory insurance is concerned, a change in the number of back orders does not affect the number of payments to general practitioners).

\section{Results}

One important goal of building a simulation model is to enable the modeler to conduct 'whatif' policy experiments with the computer model. Many modelers consider this as the only useful objective. As various authors have pointed out however, building a conceptual model often generates very useful policy relevant information (Eden et al., 1983; Meadows, 1989; Wolstenholme, 1982; Wolstenholme and Coyle, 1983).
In our case several tangible results materialized from this conceptual model-building stage. These are related to the quality of the conceptual model, the definition of the policy problem and the structuring of future research efforts in health care processes.

In our view, the quality of the conceptual model was increased drastically on a number of aspects. First, with regard to the number of variables included in the model. This number increased considerably during the knowledge elicitation stage. Our preliminary model contained about 40 variables and the final model contains more than 80 . Although a larger conceptual model is not necessarily better, the increase was primarily caused by refinement of the concepts and relationships in the preliminary model. We consider that as an important improvement of the quality of the model. We have presented several examples in this article indicating that concepts used in the research literature and in discussions about health care are frequently too ambiguous. Concepts like workload, general practitioner's view of his job and uncertainty of a general practitioner were refined considerably. We have also presented examples of refinement of relationships, sometimes identifying new feedback loops, during the process of knowledge elicitation (see also Vennix et al., 1990).

The model-building process also had an impact on the definition of the policy problem of cost reduction. Before starting the model-building process various persons were quite convinced that the best way to cut health care costs would be to reduce the number of referrals by general practitioners. This seems obvious since transactions by medical specialists are much more expensive than those by general practitioners, particularly if patients are admitted into hospital. During the model-building process it became clear that there are feedback processes which might counteract the cost reduction effect from the reduction of the number of referrals. For instance, through an increase in the number of transactions by medical specialists to compensate for the loss of new patients. To gain more insight in these feedback processes the conceptual model was formalized. Preliminary analyses, however, seem to indicate that cost reduction effects do occur when reducing the number of referrals (Verburgh et al., 1990). Future analyses with a 
more elaborated model will have to provide a more final answer to this question.

A third result which materialized relates to further empirical research into health care processes. As we have stated, the discussions in the workshop showed that various parts of the system are ill-understood. As a result a research project has been started aimed at filling in the gaps in knowledge on the factors determining back orders by general practitioners.

\section{Summary and discussion}

In this article we have concentrated on a structured approach to knowledge elicitation for conceptual model building. The approach basically employs an iterative sequence of ConceptualizeFeedback-Talk. We suggested that a three-step approach using different kinds of data collection methods is an appropriate way to structure the knowledge elicitation process. A questionnaire was used to have a number of experts $(60)$ comment on binary relationships of a preliminary model designed by the project group. Next a workbook was employed to have a subset (18) of the first group of experts criticize a number of more complex submodels. Finally, in a structured workshop participants were put in a position to discuss these submodels in more detail.

At the beginning of this article we indicated that our primary aim was to develop and implement an alternative procedure for knowledge elicitation in conceptual model building. We did not explicitly aim at systematically and objectively evaluating this procedure. As stated this is the subject for future research efforts. One obvious way to evaluate it would be to compare it to the 'traditional' way of the system dynamics approach in knowledge elicitation, i.e. by interviews and group discussions. Without trying to be exhaustive one might think of the following criteria for evaluation:

- The quality of the resulting conceptual model.

- Time investment of the participants.

- Satisfaction of participants with the process.

- Model acceptance and reduction of disagreement.

- The degree to which it improves insight into the problem and generates new solutions.
Although we did not carry out an objective evaluation of this kind, we have indications that the procedure did score well on some of the above criteria. One of these is the time investment. The main reason to use a preliminary model and this approach was to cut on the time investment of participants. In sum it took participants in this procedure about 8 to 10 hours to complete the questionnaires, to fill out the workbook and to participate in the workshop. This seems lower than in most cases where one starts from scratch. Morecroft et al. (1989, p. 3) for instance report an average time investment by the client of $2 \frac{1}{2}$ days. On the other hand it might well be the case that by using a preliminary model the project group has a great deal of influence on the design of the conceptual model. This might for instance decrease the feeling of ownership over the model for the client. This in turn could affect model acceptance and the actual use of the model in the organization once the model builders have left. Clearly this is one of the topics which needs to be studied more carefully in the future.

Another reason to use this approach was to match the various tasks in model building with individual and group work. Apart from lower time investment the procedure allows participants to do several tasks individually at home and to join together once it is clear what the exact topics are which need to be discussed. In that sense the procedure proved to be very valuable, particularly in our case where there were general practitioners in our sample who really lack the time to attend meetings of this sort.

As indicated the quality of the conceptual model clearly increased and the client seems to be rather satisfied with the whole project. This can be deduced from the fact that the client organization provided additional financial support to write a book on the project. Moreover, it supports our continuing efforts to formalize the model and to design a flexible computer-based learning environment in which health care planners and workers can conduct policy experiments with the model themselves.

Positive reactions on the project were also obtained during a conference attended by almost 200 persons from the health care field (e.g general practitioners, scientists, health care planners, modelers). During this conference we had a number of presentations on the project and a panel 
discussion. At the end of the conference participants filled out a questionnaire. Two thirds considered the subjects that were presented interesting to very interesting on a five-point scale ranging form very uninteresting to very interesting. 24 Persons were very interested and 35 interested (again on a five-point scale) in talking to the project group about the applicability of this model-building approach for their own organization. As a result thus far one new model-building project on the organization of home care in the future has been started. This project will use a procedure similar to the one described in this article.

In sum, the whole project, including a similar approach to identify feasible policy options and formalize part of the conceptual model, took several years to complete (Vennix et al., 1990). Calendar time for the procedure described in this article took a little over one year. This was caused by two factors. First, the project group had to design and test various novel procedures (e.g. questionnaire, workbook). Second, the project group did not work full time on it. We estimate that, given our experience with this approach, it should be possible to finish a conceptual modeling phase using this approach in about three to six months.

At the moment we have already formalized and quantified part of the model, i.e the patients flow model and the costs involved. Our efforts are now aimed at including a number of influencing factors on this patients flow model. In addition we have designed a computer-based learning environment in which health care policy makers can conduct a number of policy experiments with the computer model 1) to increase their systemic thinking about the subject, 2) to improve communication about health care cost reduction and 3 ) to stimulate their insight into potential effects of options aiming at cost reduction.

\section{Acknowledgments}

The authors wish to thank Doeke Post and Henk Poppen for their participation in the project and Loes Builtjes, Jan Faber, George Richardson and Wim Scheper for valuable comments on earlier versions of this article.

\section{References}

Bouchard, T. (1969), "Personality, problem solving procedure and performance in small groups", Journal of Applied Psychology 53, 1-29.

Bouchard, T. (1972), "A comparison of two group brainstorming procedures", Journal of Applied Psychology 56, 418421.

Dalkey, N. (1969), "An experimental study of group opinion: The Delphi method", Futures 1, 408-426.

Delbecq, A.L., Van de Ven, A.H., and Gustafson, D.H. (1975), Group Techniques for Program Planning: A Guide to Nominal Group and Delphi Processes, Scott, Foresman, Glenview, IL.

Diehl, M., and Stroebe, W. (1987), "Productivity loss in brainstorming groups: Toward the solution of a riddle", Journal of Personality and Social Psychology 53, 497-509.

Dijk, J.A.G.M. van (1990), "Delphi questionnaires versus individual and group interviews: a comparison case", Technological Forecasting and Social Change 37, 293-304.

Duke, R.D. (1980), “A paradigm for game design”, in: C.S. Greenblat and R.D. Duke, Principles and Practices of Gaming-Simulation, Sage, London, 63-72.

Eden, C. (1985), "Perish the thought", Journal of the Operational Research Society 36, 809-819.

Eden, C., Jones, S., and Sims, D. (1983), Messing About in Problems: An Informal Structured Approach to Their Identification and Management, Pergamon Press, New York.

Fischer, G.W. (1981), "When oracles fail: A comparison of four procedures for aggregating subjective probability forecasts", Organizational Behavior and Human Performance 28, 96-110.

Forrester, J.W. (1987), "Lessons from system dynamics modeling", System Dynamics Review 3, 136-149.

Forrester, J.W. (1991), "Policies, decisions and information sources for modeling, European Journal of Operational Research 59, 42-63, this issue.

Geus, A. de (1988), "Planning as learning", Harvard Business Review, March-April, 70-74.

Grünwald, C.A. (1987), Beheersing van de gezondheidszorg, VUGA, Den Haag.

Gustafson, D.H., Shukla, R.K., Delbecq, A., and Walster, G.W. (1973), "A comparative study of differences in subjective likelihood estimates made by individuals, interacting groups, delphi groups and nominal groups", Organizational Behavior and Human Performance 9, 280-291.

Hart, S.L., Boroush, M., Enk, G., and Hornick, W. (1985), "Managing, complexity through consensus mapping: Technology for the structuring of group decisions", Academy of Management Review 10, 587-600.

House, P.W. (1982), The Art of Public Policy Analysis: The Arena of Regulations and Resources, Sage, London.

Lamm, H., and Trommsdorf, G. (1973), "Group versus individual performance on tasks requiring ideational proficiency (brainstorming): A review", European Journal of Social Psychology 3, 361-388.

Linstone, H., and Turoff, M. (1975), The Delphi Method: Techniques and Applications, Wiley, New York.

Mason, O.M., and Mitroff, I.I. (1981), Challenging Strategic Planning Assumptions: Theory, Cases and Techniques, Wiley, New York. 
Meadows, D.H. (1989), "System dynamics meets the press", System Dynamics Review 5, 69-80.

Meadows, D.H., and Robinson, J.M. (1985), The Electronic Oracle: Computer Models and Social Decisions, Wiley, Chichester, UK.

Mintzberg, H. (1990), "The manager's job: Folklore and fact", Harvard Business Review, March-April, 163-176.

Morecroft, J.D.W., Lane, D.C., and Viita, P.S. (1989), “Modeling growth strategy in a biotechnology startup firm", Working Paper GS-32-89, London Business School, also published by Shell International Petroleum Company Ltd., "Group planning as strategic planning in Shell", Series No. 9, May 1990, Shell Centre, London SE1 7NA. UK, and forthcoming in the System Dynamics Review, 1991.

Nelms, K.R., and Porter, A.L. (1985), "EFTE: an interactive delphi method", Technological Forecasting and Social Change 28, 43-61.

Poppen, H.J. (1987), "In den eersten lijn gemeeten, een systeemdynamische benadering van de huisartsgeneeskunde", MsC. Thesis, State University of Groningen.

Randers, J. (1977), "The potential in simulation of macro social processes: or how to be a useful builder of simulation models", Grupper for Ressursstudier, Oslo.

Reagan-Cirincione, P. (1991), "Improving the accuracy of forecasts: A process intervention combining social judgment analysis and group facilitation", Ph.D. Dissertation, Rockefeller College of Public Affairs and Policy, State University of New York at Albany, NY.

Richardson, G.P., and Pugh, A.L. (1981), Introduction to System Dynamics Modeling with DYNAMO, MIT Press, Cambridge, MA.

Richardson, G.P., and Senge, P.M. (1989), "Corporate and statewide perspectives on the liability insurance crisis", in: P.M. Milling and E.O. Zahn (eds.), Computer-based Management of Complex Systems, Proceedings of the 1989 International Conference of the System Dynamics Society, Springer-Verlag, Berlin, 442-457.

Richmond, B. (1987), "The Strategic Forum: From vision to strategy to operating policies and back again", High Performance Systems, Lyme, NH.

Rohrbaugh, J. (1979), "Improving the quality of group judgment: Social Judgment Analysis and the Delphi technique", Organizational Behavior and Human Performance 24, 73-92.

Rohrbaugh, J. (1981), "Improving the quality of group judgment: Social Judgment Analysis and the Nominal Group
Technique", Organizational Behavior and Human Performance 28, 272-288.

Sackman, H. (1975), Delphi Critique: Expert Opinion, Forecasting and Group Process, Heath, Lexington, MA.

Sniezek, J.A. (1990), "A comparison of techniques for judgmental forecasting by groups with common information", Group \& Organization Studies 15, 5-19.

Stenberg, L. (1980), "A modeling procedure for public policy", in: J. Randers, (ed.), Elements of the System Dynamics Method, MIT Press, Cambridge, MA, 292-312.

Stewart, T.R. (1987), "The delphi technique and judgmental forecasting", Climatic Change 11, 97-113.

Underwood, S.E. (1984), "An evaluation of a participative technique for strategic planning”, unpublished paper, University of Ann Arbor, MI.

Van de Ven, A.H., and Delbecq, A.L. (1974), "The effectiveness of nominal, delphi and interacting group decision making processes". Academy of Management Journal 17 , 605-621.

Vennix, J.A.M., (1990), Mental models and computer models: Design and evaluation of a computer-based learning environment for policy making, Ph.D. dissertation. University of Nijmegen.

Vennix, J.A.M., and Geurts, J.L.A. (1987), "Communicating insights from complex simulation models: A gaming approach", Simulation and Games 18, 321-343.

Vennix, J.A.M., Gubbels, J.W., Post, D., and Poppen, H.J. (1990), "A structured approach to knowledge elicitation in conceptual model-building", System Dynamics Review 6, 194-208.

Verburgh, L.H., Gubbels, J.W., Vennix, J.A.M., and Post, D. (1990), "Model-based analyses of the Dutch Health Care System", in: D.F. Andersen, G.P. Richardson and J.D. Sterman, Proceedings of the 1990 International System Dynamics Conference, Chestnut Hill, MA, July 10-13, 12111225.

Weil, H.B. (1980), "The evolution of an approach for achieving implemented results from System Dynamics projects", in: J. Randers, J. (ed.), Elements of the System Dynamics Method, MIT Press, Cambridge, MA, 271-291.

Wolstenholme, E.F. (1982), "System Dynamics in perspective", Journal of the Operational Research Society 33, 547-556.

Wolstenholme, E.F., and Coyle, R.G. (1983), "The development of system dynamics as a methodology for system description and qualitative analysis", Journal of the Operational Research Society 34, 569-581. 